\title{
ON A CONJECTURE OF P. PUCCI AND J. SERRIN
}

\author{
Hans-Christoph Grunau
}

Received:

AMS-Classification (1991): 35J65, 35J40

We are interested in the critical behaviour of certain dimensions in the semilinear polyharmonic eigenvalue problem

$$
\begin{cases}(-\Delta)^{m} u=\lambda u+|u|^{s-1} u, u \not \equiv 0 & \text { in } B \\ D^{\alpha} u \mid \partial B=0 & \text { for }|\alpha| \leq m-1 .\end{cases}
$$

Here $m \in \mathbb{N}, B \subset \mathbb{R}^{n}$ is the unit ball, $n>2 m, \lambda \in \mathbb{R} ; s=(n+2 m) /(n-2 m)$ is the critical Sobolev exponent.

Due to the criticality of the exponent $s$, a nontrivial solution to (1) may exist at most for $\lambda>0$, if $m=1([9])$, and for $\lambda \geq 0$, if $m \in \mathbb{N}([10])$.

The case $m=1$ has been studied extensively by Brezis and Nirenberg [4] because it serves as a model problem for other important variational problems with critical growth as the Yamabe problem or the $H$-system. They discovered an interesting phenomenon: There exists a positive radial solution to (1) for every $\lambda \in\left(0, \Lambda_{1}\right)$, if $n \geq 4$, and for every $\lambda \in\left(\frac{1}{4} \Lambda_{1}, \Lambda_{1}\right)$, if $n=3$. In the latter case problem (1) has no nontrivial radial solution, if $\lambda \leq \frac{1}{4} \Lambda_{1}$. Here $\Lambda_{m}$ denotes the first eigenvalue of $(-\Delta)^{m}$ in $B$ under homogeneous Dirichlet boundary conditions (1.b).

Pucci and Serrin [11] raised the question in which way this critical behaviour of certain dimensions depends on the order $2 m$ of the semilinear polyharmonic eigenvalue problem (1), if $m$ increases arbitrarily. In order to have a suggestive name for those dimensions we define according to Pucci and Serrin [11]:

DEFINITION 1: The dimension $n$ is called critical (with respect to the boundary value problem (1)) iff there is a positive bound $\Lambda>0$ such that a necessary condition for a nontrivial radial solution to (1) to exist is

$$
\lambda>\Lambda .
$$

Pucci and Serrin [11] showed that for any $m$ the dimension $n=2 m+1$ is critical and, moreover, that $n=5,6,7$ are critical in the fourth order problem, $m=2$. They conjectured: 
CONJECTURE OF PUCCI AND SERRIN: The critical dimensions for the boundary value problem (1) are precisely $n=2 m+1, \ldots, 4 m-1$.

By the work of Brezis and Nirenberg $[\mathrm{BrN}]$ this conjecture is true for $m=1$. Combining the result of Pucci and Serrin [11] with existence theorems of Edmunds, Fortunato, Jannelli [5] and Noussair, Swanson, Yang Jianfu [8] we see that the conjecture is also true for $m=2$. The author [6] has shown existence of positive solutions. We remark, that in higher order equations positivity properties are more subtle than in second order equations. We quote from $[6]$ :

THEOREM 2: Let $n>2 m, s=(n+2 m) /(n-2 m), B \subset \mathbb{R}^{n}$ the unit ball. Let $\Lambda_{m}$ denote the first Dirichlet eigenvalue of $(-\Delta)^{m}$ in $B$.

a) If $n \geq 4 m$, then for every $\lambda \in\left(0, \Lambda_{m}\right)$ there exists a positive radial solution $u \in C^{\infty}(B) \cap$ $C^{2 m+1}(\bar{B})$ to the Dirichlet problem (1). $u=u(r)$ strictly decreases in $r=|x| \in(0,1)$.

b) If $2 m+1 \leq n \leq 4 m-1$, then there exists a $\bar{\Lambda}=\bar{\Lambda}(n, m) \in\left(0, \Lambda_{m}\right)$ such that for every $\lambda \in\left(\bar{\Lambda}, \Lambda_{m}\right)$ the Dirichlet problem (1) has a solution $u$ as above.

In particular the critical dimensions are at most $n=2 m+1, \ldots, 4 m-1$. Moreover, Bernis and the author [2], [7] have proven the Pucci-Serrin conjecture for $m=3$ and $m=4$; for any $m \geq 5$ there are at least five critical dimensions $n=2 m+1, \ldots, 2 m+5$. But in general the conjecture of Pucci and Serrin is still open.

In Theorem 2 we additionally have positivity of solutions. In order to show that this theorem is optimal in so far as for $n=2 m+1, \ldots, 4 m-1, \bar{\Lambda}$ cannot be replaced by 0 , we do not need to show the original Pucci-Serrin conjecture. It suffices to exclude positive radial solutions for $\lambda$ close to 0 .

DEFINITION 3: The dimension $n$ is called weakly critical (with respect to the boundary value problem (1)) iff there is a positive bound $\tilde{\Lambda}>0$ such that a necessary condition for a positive radial solution to (1) to exist is

$\lambda>\tilde{\Lambda}$

THEOREM 4: The weakly critical dimensions for the semilinear polyharmonic eigenvalue problem (1) are precisely $n=2 m+1, \ldots, 4 m-1$.

PROOF: We assume that $n \in\{2 m+1, \ldots, 4 m-1\}$ and that there exists a positive radial solution $u \in C^{2 m}(\bar{B})$ to (1). Then from [10] we know that necessarily

(2) $\quad \lambda \geq 0$.

From the differential equation (1) we immediately conclude

(3) $(-\Delta)^{m} u>0$ in $B$.

In what follows only radial functions are considered. So we write $r=|x|, u=u(r), u^{\prime}(r)=$ $\frac{d u}{d r}(r)=\sum_{i=1}^{n} u_{x_{i}}(|x|) \cdot \frac{x_{i}}{|x|}$. 
1. We introduce the positive weight function $w(r):=\left(1-r^{2}\right)^{m-1}$. With help of the simple observations:

$$
\left\{\begin{array}{l}
(-\Delta)^{m} w=0 \\
\left.\left(\frac{d}{d r}\right)^{j} w\right|_{r=1}=0 \text { for } j=0, \ldots, m-2 \\
\left.\left(-\frac{d}{d r}\right)^{m-1} w\right|_{r=1}>0
\end{array}\right.
$$

integration by parts yields, if $m$ is even:

$$
\begin{aligned}
& \int_{B} w(-\Delta)^{m} u d x=\sum_{i=1}^{n} \int_{B} \frac{\partial}{\partial x_{i}}\left((-\Delta)^{(m / 2)-1} w\right) \cdot \frac{\partial}{\partial x_{i}}\left((-\Delta)^{m / 2} u\right) d x \\
& \quad=(-1)^{m-1} \int_{\partial B}\left(\frac{d}{d r}\left(\Delta^{(m / 2)-1} w\right)\right)\left(\Delta^{m / 2} u\right) d \omega=C(n, m) \int_{\partial B} \Delta^{m / 2} u d \omega
\end{aligned}
$$

and if $m$ is odd:

$$
\begin{aligned}
& \int_{B} w(-\Delta)^{m} u d x=\int_{B}\left((-\Delta)^{(m-1) / 2} w\right) \cdot\left((-\Delta)^{(m+1) / 2} u\right) d x \\
& =(-1)^{m} \int_{\partial B}\left(\Delta^{(m-1) / 2} w\right)\left(\frac{d}{d r} \Delta^{(m-1) / 2} u\right) d \omega \\
& =C(n, m) \int_{\partial B}\left(-\frac{d}{d r} \Delta^{(m-1) / 2} u\right) d \omega .
\end{aligned}
$$

From (4) we see that the constants $C(n, m)$ are strictly positive. Applying the CauchySchwarz inequality we find

$$
\left(\int_{B} w(-\Delta)^{m} u d x\right)^{2} \leq \begin{cases}C(n, m) \int_{\partial B}\left(\Delta^{m / 2} u\right)^{2} d \omega, & \text { if } m \text { is even } \\ C(n, m) \int_{\partial B}\left(\frac{d}{d r} \Delta^{(m-1) / 2} u\right)^{2} d \omega, & \text { if } m \text { is odd }\end{cases}
$$

2. The crucial information, which we take from the Dirichlet problem (1), is the following "Pohožaev-type" identity:

(6) $2 m \lambda \int_{B} u^{2} d x= \begin{cases}\int_{\partial B}\left(\Delta^{m / 2} u\right)^{2} d \omega, & \text { if } m \text { even, } \\ \int_{\partial B}\left(\frac{d}{d r} \Delta^{(m-1) / 2} u\right)^{2} d \omega, & \text { if } m \text { odd. }\end{cases}$

This identity is due to Pohožaev [9] for $m=1$ and to Pucci and Serrin [10] for $m \in \mathbb{N}$. It is deduced from the equation (1) by means of the testing functions $r u^{\prime}$ and $u$ itself and by partial integration. Combining (5) and (6) we have:

$$
\left(\int_{B} w(-\Delta)^{m} u d x\right)^{2} \leq C(n, m) \cdot \lambda \int_{B} u^{2} d x .
$$


3. We want to show that the weighted $L^{1}$-norm $\int_{B} w(-\Delta)^{m} u d x$ is equivalent to the $L^{1}$-norm $\int_{B}(-\Delta)^{m} u d x=\int_{B}\left|(-\Delta)^{m} u\right| d x$, cf. also (3). For this purpose we need some additional property of $(-\Delta)^{m} u$.

Because we assume the solution to be positive, we have the differential inequality (3). Moreover $u$ is supposed to be radial, with help of [12, Prop. 1] we conclude that $u=u(r)$ strictly decreases in $r \in(0,1)$. This can also be shown by elementary calculus: polar form of $(-\Delta)$, mean value theorem, counting zeros. Looking again at the differential equation (1) and observing (2), we see that also $\left((-\Delta)^{m} u\right)(r)$ strictly decreases in $r \in(0,1)$. Employing this monotonicity we may estimate as follows:

$$
\begin{aligned}
0 & <\int_{B}(-\Delta)^{m} u d x=\int_{|x| \leq 1 / 2}(-\Delta)^{m} u d x+\int_{1 / 2 \leq|x| \leq 1}(-\Delta)^{m} u d x \\
& \leq \int_{|x| \leq 1 / 2}(-\Delta)^{m} u d x+\int_{1 / 2 \leq|x| \leq 1} d x \cdot\left(\left.(-\Delta)^{m} u\right|_{r=1 / 2}\right) \\
& \leq \int_{|x| \leq 1 / 2}(-\Delta)^{m} u d x+2^{n} \int_{|x| \leq 1 / 2} d x \cdot\left(\left.(-\Delta)^{m} u\right|_{r=1 / 2}\right) \\
& \leq\left(2^{n}+1\right) \int_{|x| \leq 1 / 2}(-\Delta)^{m} u d x \leq\left(2^{n}+1\right)\left(\frac{4}{3}\right)^{m-1} \int_{|x| \leq 1 / 2} w(-\Delta)^{m} u d x \\
\Rightarrow 0 & <\int_{B}(-\Delta)^{m} u d x \leq C(n, m) \int_{B} w(-\Delta)^{m} u d x .
\end{aligned}
$$

Together with (7) we come up with

$$
\left\|(-\Delta)^{m} u\right\|_{L^{1}}^{2} \leq \lambda \cdot C(n, m)\|u\|_{L^{2}}^{2} .
$$

4. It remains to estimate the $L^{1}$-norm of $(-\Delta)^{m} u$ from below by the $L^{2}$-norm of $u$ itself. We use a duality argument, which was proposed by Brezis and Nirenberg [4, Theorem 1.2"]. Let $\varphi \in W^{2 m, 2}(B) \cap W_{0}^{m, 2}(B)$ be a solution of $(-\Delta)^{m} \varphi=u$. As $n<4 m$, we have a continuous embedding $W^{2 m, 2}(B) \hookrightarrow C^{0}(\bar{B})$. By means of elliptic estimates [1] we find:

$$
\begin{aligned}
\|\varphi\|_{C^{0}} & \leq C\|\varphi\|_{W^{2 m, 2}} \leq C\left\|(-\Delta)^{m} \varphi\right\|_{L^{2}}=\|u\|_{L^{2}} ; \\
\|u\|_{L^{2}}^{2} & =\int_{B} u \cdot u d x=\int_{B}(-\Delta)^{m} \varphi \cdot u d x=\int_{B} \varphi \cdot(-\Delta)^{m} u d x \\
& \leq\|\varphi\|_{C^{0}} \cdot\left\|(-\Delta)^{m} u\right\|_{L^{1}} \leq C\|u\|_{L^{2}} \cdot\left\|(-\Delta)^{m} u\right\|_{L^{1}} ; \\
\|u\|_{L^{2}}^{2} & \leq C(n, m)\left\|(-\Delta)^{m} u\right\|_{L^{1}}^{2} .
\end{aligned}
$$

Combining this estimate with (8) we finally have

$$
\lambda \geq \frac{1}{C(n, m)}>0
$$

as stated.

REMARK: It seems that a full proof of the original Pucci-Serrin conjecture, if possible at all, will be significantly more difficult.

The most general question in this context, whether we have nonexistence of any nontrivial (not necessarily radial) solution to (1) for $\lambda$ close to 0 , is completely open even in the second order case $(m=1)$, as far as the author knows. 


\section{References}

1. Sh. Agmon, A. Douglis, L. Nirenberg, Estimates near the boundary for solutions of elliptic partial differential equations satisfying general boundary conditions. I, Commun. Pure Appl. Math. 12, 623-727 (1959)

2. F. Bernis, H.-Ch. Grunau, Critical exponents and multiple critical dimensions for polyharmonic operators, J. Differ. Equations 117, 469-486 (1995)

3. T. Boggio, Sulle funzioni di Green d'ordine m, Rend. Circ. Mat. Palermo 20, 97-135 (1905)

4. H. Brezis, L. Nirenberg, Positive solutions of nonlinear elliptic equations involving critical Sobolev exponents, Commun. Pure Appl. Math. 36, 437-477 (1983)

5. D. E. Edmunds, D. Fortunato, E. Jannelli, Critical exponents, critical dimensions and the biharmonic operator, Arch. Rational Mech. Anal. 112, 269-289 (1990)

6. H.-Ch. Grunau, Positive solutions to semilinear polyharmonic Dirichlet problems involving critical Sobolev exponents, Calc. Var. 3, 243-252 (1995)

7. H.-Ch. Grunau, Critical exponents and multiple critical dimensions for polyharmonic operators. II, Boll. Unione Mat. Ital. (7) 9-B, 815-847 (1995)

8. E. S. Noussair, Ch. A. Swanson, Yang Jianfu, Critical semilinear biharmonic equations in $\mathbb{R}^{N}$, Proc. Royal Soc. Edinburgh 121 A, 139-148 (1992)

9. S. I. Pohožaev, Eigenfunctions of the equation $\Delta u+\lambda f(u)=0$, Soviet Math. Dokl. 6, 1408-1411 (1965)

10. P. Pucci, J. Serrin, A general variational identity, Indiana Univ. Math. J. 35, 681-703 (1986)

11. P. Pucci, J. Serrin, Critical exponents and critical dimensions for polyharmonic operators, J. Math. Pures Appl. 69, 55-83 (1990)

12. R. Soranzo, A priori estimates and existence of positive solutions of a superlinear polyharmonic equation, Dyn. Syst. Appl. 3, 465-487 (1994)

13. Ch. A. Swanson, The best Sobolev constant, Applicable Anal. 47, 227-239 (1992)

Fachgruppe Mathematik, Universität Bayreuth, 95440 Bayreuth, Germany 